\title{
Electron-vortex separation in quantum dots
}

\author{
E. Anisimovas, ${ }^{1,2}$ M. B. Tavernier, ${ }^{2}$ and F. M. Peeters ${ }^{2}$ \\ ${ }^{1}$ Department of Theoretical Physics, Vilnius University, Sauletekio Avenue 9, LT-10222 Vilnius, Lithuania \\ ${ }^{2}$ Departement Fysica, Universiteit Antwerpen, Groenenborgerlaan 171, B-2020 Antwerpen, Belgium
}

(Received 14 May 2007; published 24 January 2008)

\begin{abstract}
We calculate the many-body wave function structure of a strongly correlated electron system in a quantum dot placed into a magnetic field. The exact numerical results show the formation of electron-vortex complexes in accordance with the composite fermion picture. We show that the electron-vortex distance is a universal function shared by various vortex shells and quantum dots containing different numbers of electrons. This distance is a quadratic function of the filling factor. In contrast, the approximate rotating-electron-molecule approach fails to capture this universality and predicts an electron-vortex distance linear in the filling factor.
\end{abstract}

DOI: 10.1103/PhysRevB.77.045327

PACS number(s): 73.21.La, 71.10.-w

\section{INTRODUCTION}

Successful identification of quasiparticles that faithfully represent collective states of matter has been a topic of utmost importance in condensed matter physics. One of the recent searches for the right quasiparticle was prompted by the discovery of the fractional quantum Hall effect, ${ }^{1}$ and the intricate pattern of fractional filling factors at which the effect is observed was elegantly accounted for in terms of the composite fermion $(\mathrm{CF})^{2,3}$ picture that may intuitively be interpreted as bound states of an electron plus an even number of magnetic vortices. ${ }^{4}$

This CF phase is an intermediate one. At weaker magnetic fields, corresponding to integer filling factors, one recovers the perturbative physics of a weakly interacting electron liquid. On the other hand, at stronger magnetic fields, corresponding to the inverse fractional filling factors around $\nu$ $\approx \frac{1}{7}-\frac{1}{9}$ and below, 5,6 the quantum-fluid ground state is replaced by an essentially classical Wigner crystal. ${ }^{7}$

The same sequence of states must also be present in confined systems, i.e., quantum dots. ${ }^{8}$ Here, due to the finite number of particles, it is possible to try and to solve the complete many-body problem by performing exact diagonalizations. ${ }^{9-13}$ Although this method is confronted by insurmountable computational difficulties when the system size and interaction strength increase, an accurate treatment of, say, $N=6$ particles is well within reach.

The CF theory has been successfully applied to describe the electron states in quantum dots. ${ }^{3,14}$ Alternatively, it was proposed that strongly correlated quantum dots may be visualized as collectively rotating-electron molecules (REMs). In the strong magnetic field limit, electrons tend to crystallize into a Wigner crystallite composed of concentric polygonal electron rings. The electronic charge distribution may be approximated by Gaussian functions centered at the positions of the electrons, and the circular symmetry is restored by projection techniques. In this manner, analytic wave functions were derived ${ }^{15}$ and shown to provide a more accurate description ${ }^{16}$ than the $\mathrm{CF}$ formalism, in particular, in the relevant filling factor range of $\frac{1}{9} \leqslant \nu \leqslant \frac{1}{5}$.

However, the composite fermion picture was able to restore its credibility by resorting to perturbative schemes based on the mixing of quasi-Landau levels. ${ }^{17}$ These calcu- lations bear increased computational costs and produce lower ground state energies, and close to unity overlaps with the exact wave functions. When applied to extended systems, the perturbative CF schemes also predict lower energies associated with fractional quantum Hall states and the possible persistence of this effect down to the ultralow filling factor $\nu=\frac{1}{31}$.

The comparison of the two different approaches (as well as the prevalence of the different nature of the ground state) has thus been mostly confined to the comparison of their energies and overlaps of the exact and approximate wave functions, which is global and integral characteristics. Attention to the details was limited to orbital occupations and charge density correlation functions.

The purpose of this paper is to cast light on the issue of formation of complex entities in quantum dots. Exactdiagonalization studies reveal the emergence of structures that consist of an electron and an even number of nearby vortices and thus have the same character as the composite fermions. We investigate the relative distribution of electrons and vortices and uncover an important universality in the behavior of the electron-vortex distances. This universality, present in the exact-diagonalization calculations, is, however, absent from the REM results.

Our paper is organized in the following way. In Sec. II, the computational model is described, and in Sec. III, the results are given and contrasted to the REM predictions. In Sec. IV, we give our conclusions.

\section{COMPUTATIONAL MODEL}

We consider a parabolic quantum dot populated by $N$ $=3-6$ electrons in a magnetic field. The Hamiltonian is written as

$$
H=\sum_{i=1}^{N}\left[\frac{1}{2 m}\left(\boldsymbol{p}_{i}+\frac{e}{c} \boldsymbol{A}_{i}\right)^{2}+\frac{1}{2} m \omega_{0}^{2} r_{i}^{2}\right]+\sum_{i<j} \frac{e^{2} / \boldsymbol{\epsilon}}{\left|\boldsymbol{r}_{i}-\boldsymbol{r}_{j}\right|} .
$$

Here, $\boldsymbol{A}_{i}$ is the usual symmetric-gauge vector potential at the position of the $i$ th electron, $\omega_{0}$ denotes the confinement frequency, and the medium parameters are the effective electron mass $m$ and the dielectric constant $\epsilon$. The number of parameters is greatly reduced by switching to dimensionless units, 
whereby the energies are measured in $\hbar \omega_{0}$ and lengths in $l_{0}=\sqrt{\hbar / m \omega_{0}}$. A convenient measure of the magnetic field strength is the ratio of the cyclotron and confinement frequencies $\gamma=\omega_{c} / \omega_{0}$, and the effective Coulomb interaction strength is $\lambda=l_{0} / a_{B}$, with $a_{B}$ being the effective Bohr radius. The Hamiltonian is thus transformed into

$$
H(\lambda, \gamma)=\sum_{i=1}^{N}\left[-\frac{\nabla_{i}^{2}}{2}+\left(1+\frac{\gamma^{2}}{4}\right) \frac{r_{i}^{2}}{2}\right]+\frac{\gamma L}{2}+\sum_{i<j}^{N} \frac{\lambda}{\left|\boldsymbol{r}_{i}-\boldsymbol{r}_{j}\right|} .
$$

Here, $L$ denotes the angular momentum, either an operator or the quantum number of the corresponding rotationally symmetric solution.

Note that we disregard the spin degree of freedom. If a finite Zeeman splitting is present, at high magnetic fields, the ground states will be fully polarized. This fact only sets limitations on the possible values of the angular momentum $L$ in a ground state. As soon as the magnetic field is sufficiently strong to establish spin polarization, in a three-electron quantum dot, these values follow the sequence of multiples of the electron number, ${ }^{18}$ which is, $L=6,9,12,15, \ldots$. A similar pattern of multiples is observed in a five-electron quantum $\operatorname{dot}^{13}-L=15,20,25, \ldots$. In a four-electron quantum dot, the angular momentum values follow a shifted sequence, ${ }^{10} \mathrm{~L}$ $=10,14,18, \ldots$, while in a six-electron quantum dot, one encounters $^{13}$ a competition between two configurations (a hexagonal electron ring and a pentagonal ring surrounding a central electron). Thus, the sequence of angular momentum values in spin-polarized ground states is intertwined, ${ }^{13}$

$$
L=21,25,30,35,39,40,45,50, \ldots \text {. }
$$

We observe that for a parabolic confinement, the Hamiltonian (2) may be mapped onto the Hamiltonian without a magnetic field by an appropriate scaling of coordinates,

$$
r \rightarrow r\left[1+\frac{\gamma^{2}}{4}\right]^{-1 / 4}
$$

Thus, one obtains

$$
H(\gamma, \lambda)=\left[1+\frac{\gamma^{2}}{4}\right]^{1 / 2} H\left(0, \lambda^{\prime}\right)-\frac{1}{2} \gamma L,
$$

with $\lambda^{\prime}=\lambda\left(1+\gamma^{2} / 4\right)^{-1 / 4}$.

In view of the above, we solve the zero-field Hamiltonian and obtain the results by recalculating the energies and by scaling the wave function. As in our previous work, ${ }^{11}$ we use the value $\lambda=2$ which is typical for experimentally realized quantum dots. ${ }^{19,20}$ As a matter of fact, when a strong perpendicular magnetic field is applied, the effective strength of the Coulomb repulsion with respect to the kinetic energy is set by the magnetic field strength rather than the Coulomb interaction constant. Thus, for Wigner crystallization at high magnetic fields, a large $\lambda$ is not needed, and our results are not sensitive to the choice of this parameter.

Having obtained the complete many-body wave function as a function of the coordinates of all $N$ electrons $\Psi\left(\boldsymbol{r}_{1}, \ldots, \boldsymbol{r}_{N}\right)$, we form an auxiliary quantity, further referred to as the reduced wave function. This wave function is a function of the coordinates of only one electron, while the others are pinned at certain positions $\boldsymbol{r}_{i}^{*}$,

$$
\tilde{\Psi}(\boldsymbol{r})=\Psi\left(\boldsymbol{r}, \boldsymbol{r}_{2}^{*}, \ldots, \boldsymbol{r}_{N}^{*}\right) .
$$

This quantity has proven to be a successful method of visualizing the wave function structure. While being easy to calculate, this function serves as a correlation function and encompasses the relative distributions of particles. The zeros of the reduced wave function mark the positions of the magnetic vortices. It has become a standard tool in exactdiagonalization based approaches, and it was used to calibrate the spin-density functional theory (SDFT) results. ${ }^{12} \mathrm{As}$ a matter of fact, the SDFT has the advantage of being much less complex computationally and thus can be used to treat higher particle numbers. The density-functional approach gives no (easy) access to correlation functions; however, the internal structure is displayed directly in the charge density of a broken-symmetry solution. ${ }^{8}$ It was shown that the positions of vortices in SDFT densities compare favorably to the exact-diagonalization results. ${ }^{12}$ Note, however, that SDFT vortices are not true density zeros. They are the density minima around which currents are circulating.

When evaluating the reduced wave function, we fix the $N-1$ electrons at their maximum likelihood positions. The classical equilibrium configuration is a polygonal ring, thus we place the $N$ electrons at equal distances on a ring whose radius is varied in order to obtain the maximum in $|\Psi|^{2}$. Then, $N-1$ electrons are left at their positions, while the remaining one is set to roam freely. Note that for $N=6$ quantum dots, some of the ground states have a fivefold rotational symmetry and consist of a five-electron ring plus an extra electron in the center of the dot. These ground states are easily distinguished by inspecting the corresponding charge densities. ${ }^{13}$ In these cases, we pin the central electron as well as all but one of those belonging to the ring.

We found that the position of the vortices does not depend strongly on the position of the pinned electrons as long as the electron configuration is of high probability and the vortices are bound to the electron, i.e., $\nu \leqslant \frac{1}{3}$. However, as we showed previously in Ref. 11, vortex configurations corresponding to low-probability arrangements of pinned electrons may be very different. In fact, it is even possible to induce antivortices.

\section{RESULTS}

The distribution of vortices in few-electron quantum dots was discussed in Refs. 11 and 13. Let us, however, briefly remind the reader that in a three-electron quantum dot, the vortices always appear on a straight line drawn through the positions of the electrons. When the number of electrons is four or larger, the vortices appear on rays extending from the interior of the dot through the positions of the fixed electrons and away into the exterior. The typical distribution of vortices is sketched in Fig. 1, which is obtained for $N=4$ electrons and the angular momentum $L=18$. Thus, as the magnetic field strength increases, each electron is approached by two vortices at a time, one from the interior of the dot and the 


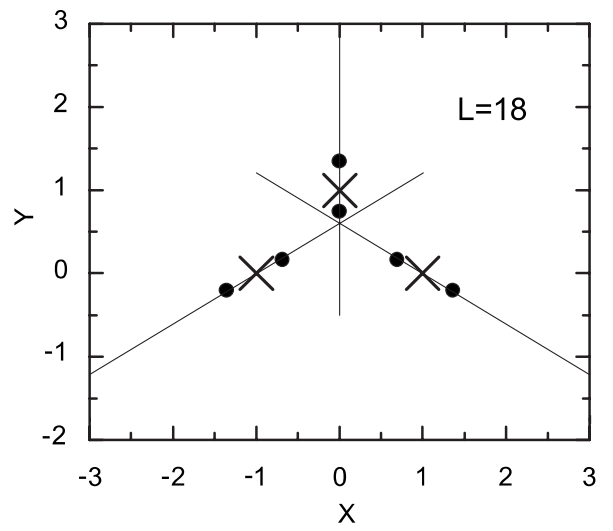

FIG. 1. Typical distribution of electrons (crosses) and vortices (dots). As an example, the ground state of the four-electron quantum dot at $L=18$ is shown.

other from the exterior. The distance from the fixed electron to the two vortices is virtually the same.

We calculate the distance $d_{\mathrm{ev}}$ between one of the electrons and the first, as well as the second nearest vortex in the direction of the dot center in quantum dots with $3 \leqslant N \leqslant 6$ electrons for a number of different $L$ values. In order to make our results more general, we plot the distances versus the filling factor $\nu$ which in quantum dots is defined by ${ }^{8}$

$$
\nu=\frac{N(N-1)}{2 L} \text {. }
$$

For a three-electron quantum dot, we used all allowed angular momenta in the interval $6 \leqslant L \leqslant 27$, thus covering the filling factor range $\frac{1}{9} \leqslant \nu \leqslant \frac{1}{2}$. The corresponding ranges for $N=4$ were $14 \leqslant L \leqslant 42$ and $\frac{1}{7} \leqslant \nu \leqslant \frac{3}{7}$, whereas for $N=5$, we covered $20 \leqslant L \leqslant 65$ and $\frac{2}{13} \leqslant \nu \leqslant \frac{1}{2}$. For a six-electron quantum dot, we used the angular momenta enumerated in Eq. (3), thus the filling factors were in the range $\frac{3}{10} \leqslant \nu \leqslant \frac{5}{7}$. The results are presented in Fig. 2 as a double logarithm plot of $d_{\mathrm{ev}} / d_{\mathrm{ee}}$, the electron-vortex distance compared to the electron-electron distance. The different symbols represent

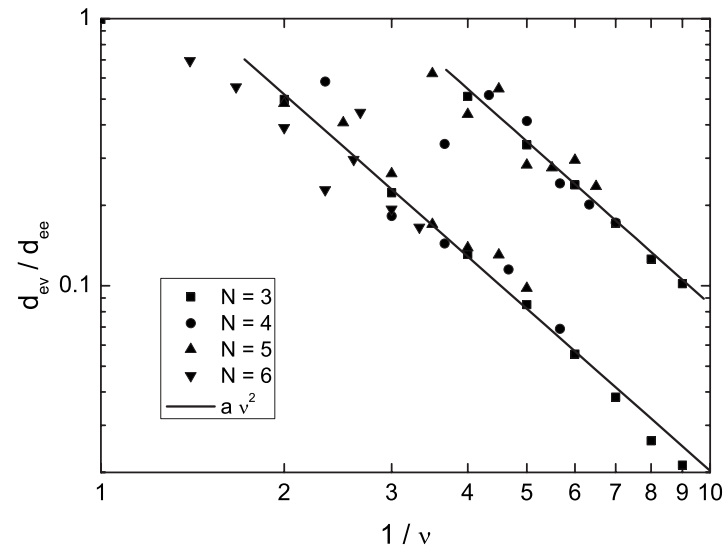

FIG. 2. The distances between an electron and the closest and the second closest vortices as a function of the inverse filling factor. The full lines denote power-law functional fits.



FIG. 3. The comparison of exact-diagonalization (solid lines) and REM (dashed and dotted lines) results in a three-electron quantum dot. The distances from a fixed electron and the two nearest vortex shells are shown. Notice that in REM, the distances to the vortices approaching from the interior (dashed lines) and exterior (dotted lines) differ significantly.

the results for different numbers of electrons. The full lines denote functional fits $d_{\mathrm{ev}} / d_{\mathrm{ee}}=a \nu^{2}$, with $a=2.10$ and 8.78 , respectively. One may clearly see that the power-law behavior is the same for all numbers of electrons and may be accurately approximated by a quadratic function of the filling factor.

This universal behavior is not present in the results extracted from the REM approach. We demonstrate this using the example of a three-electron quantum dot at filling factors down to $\nu=1 / 9$ that correspond to the angular momenta up to $L=27$. The distances between a fixed electron and the nearest two vortices approaching from the interior (exterior) of the dot are plotted in Fig. 3 with dashed (dotted) lines and compared to the exact-diagonalization results indicated by solid lines.

One sees that there is little in common between the two sets of results. REM predicts that the vortices approaching the pinned electron form the interior of the dot (dashed lines in Fig. 3) are significantly closer than their counterparts in the exterior (dotted lines in Fig. 3). This difference is decaying very slowly with magnetic field strength and persists even at extremely low filling factors. Also, the electronvortex distances demonstrate a much weaker dependence on the inverse filling factor. In fact, the asymptotical behavior is $d_{\mathrm{ev}} / d_{\mathrm{ee}} \sim \nu$, which, in the case of three electrons in a dot, may be calculated analytically since an expression for the position of vortices is explicitly known. ${ }^{11}$ These facts demonstrate that in REM, a vital part of the electron-vortex correlation is overlooked.

We make a further observation that the power-law prefactors scale approximately as $n^{2}$, with $n$ being the shell index. This behavior-as it may be seen from the following argument-is consistent with the power law $d_{\mathrm{ev}} / d_{\mathrm{ee}} \sim \nu^{2}$ and the intuitive interpretation of composite fermions as bound electron-vortex complexes. ${ }^{4}$

The number of vortices in a quantum dot is increasing proportionally to the magnetic field strength, that is, to $\nu^{-1}$. According to the composite fermion picture, the number of 
vortices found in the vicinity of a given electron (and thus forming a complex with it) must also grow linearly with $\nu^{-1}$. Now, let us denote $d_{\mathrm{ev}}(n, \nu)$ the distance to the vortices of the $n$th shell and choose a certain fraction of the interelectron distance $\alpha d_{\text {ee }}$ to represent the composite fermion radius in the sense that all the vortices sitting closer than this distance from the fixed electron are considered belonging to it. Then, the number of vortices inside this range is found by solving the equation $d_{\mathrm{ev}}(n, \nu) / d_{\mathrm{ee}}(n, \nu)=\alpha$ for the number of the shell that is just on the boundary. In order to be able to obtain the desired solution $n \sim \nu$, one must require that the function describing the distances be a function of the ratio $n / \nu$. It follows then that the dependences describing the distances as a function of the filling factor and as a function of the shell index are not independent, and the proportionality $d_{\mathrm{ev}} / d_{\mathrm{ee}}$ $\sim \nu^{2}$ implies $d_{\mathrm{ev}} / d_{\mathrm{ee}} \sim n^{2}$.

In order to verify that the observed electron-vortex complexes are indeed well defined, let us also look at the vortex distribution statistics. In contrast to our previous calculation, the radius of the electron ring is now not fixed but is allowed to have any value with probability (weighting factor) proportional to $\left|\Psi\left(\boldsymbol{r}_{1}, \ldots, \boldsymbol{r}_{N}\right)\right|^{2}$ with all the electrons placed equidistantly on a ring. The ensuing statistical distribution is depicted in Fig. 4 for two values of the filling factor in a threeelectron quantum dot. We see that as the angular momentum of the considered state increases, the probability density develops narrow peaks, thus, the vortices near the fixed electrons become well defined.

\section{CONCLUSIONS}

In conclusion, we performed a microscopic exactdiagonalization calculation with the purpose to investigate

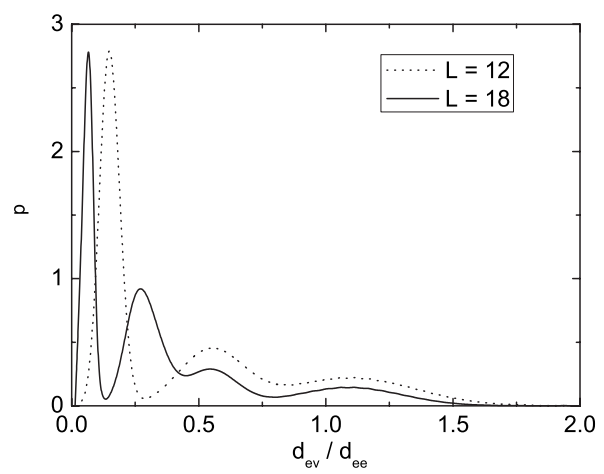

FIG. 4. The statistical distribution of electron-vortex distances in a three-electron quantum dot for two values of the angular momentum.

the structure of the many-body wave function of a strongly interacting electronic system. We were able to obtain results that provide quantitative information on the internal structure of the electron-vortex complexes forming in quantum dots. A universal, which is independent of the number of electrons, behavior of the electron-vortex distance as a function of the filling factor and the vortex shell number is disclosed. Therefore, we believe that our main result that the distance between the electron and the vortex scales as $d \sim(n / \nu)^{2}$ is also valid in an infinite two-dimensional electron gas.

\section{ACKNOWLEDGMENTS}

This work was supported by the Flemish Science Foundation (FWO-Vl), the Belgian Science Policy (IUAP), the EU Network of Excellence SANDiE, and the Lithuanian Science and Studies Foundation under Grant No. T-26/07.
${ }^{1}$ D. C. Tsui, H. L. Stormer, and A. C. Gossard, Phys. Rev. Lett. 48, 1559 (1982).

${ }^{2}$ J. K. Jain, Phys. Rev. Lett. 63, 199 (1989).

${ }^{3}$ Composite Fermions, edited by O. Heinonen (World Scientific, Singapore, 1998).

${ }^{4}$ J. K. Jain, Physica E (Amsterdam) 20, 79 (2003).

${ }^{5}$ K. Yang, F. D. M. Haldane, and E. H. Rezayi, Phys. Rev. B 64, 081301(R) (2001).

${ }^{6}$ W. Pan, H. L. Stormer, D. C. Tsui, L. N. Pfeiffer, K. W. Baldwin, and K. W. West, Phys. Rev. Lett. 88, 176802 (2002).

${ }^{7}$ E. P. Wigner, Phys. Rev. 46, 1002 (1934); C. C. Grimes and G. Adams, Phys. Rev. Lett. 42, 795 (1979).

${ }^{8}$ S. M. Reimann and M. Manninen, Rev. Mod. Phys. 74, 1283 (2002).

${ }^{9}$ See, e.g., P. A. Maksym, Phys. Rev. B 53, 10871 (1996); W. Y. Ruan and H. F. Cheung, J. Phys.: Condens. Matter 11, 435 (1999); M. Rontani, G. Goldoni, F. Manghi, and E. Molinari, Europhys. Lett. 58, 555 (2002); C. Yannouleas and U. Landman, Phys. Rev. Lett. 85, 1726 (2000); S. A. Mikhailov, Phys. Rev. B 65, 115312 (2002).
${ }^{10}$ M. B. Tavernier, E. Anisimovas, F. M. Peeters, B. Szafran, J. Adamowski, and S. Bednarek, Phys. Rev. B 68, 205305 (2003).

${ }^{11}$ M. B. Tavernier, E. Anisimovas, and F. M. Peeters, Phys. Rev. B 70, 155321 (2004).

${ }^{12}$ H. Saarikoski, A. Harju, M. J. Puska, and R. M. Nieminen, Phys. Rev. Lett. 93, 116802 (2004).

${ }^{13}$ M. B. Tavernier, E. Anisimovas, and F. M. Peeters, Phys. Rev. B 74, 125305 (2006).

${ }^{14}$ J. K. Jain and T. Kawamura, Europhys. Lett. 29, 321 (1995).

${ }^{15}$ C. Yannouleas and U. Landman, Phys. Rev. B 66, 115315 (2002).

${ }^{16}$ C. Yannouleas and U. Landman, Phys. Rev. B 68, 035326 (2003).

${ }^{17}$ G. S. Jeon, C. C. Chang, and J. K. Jain, J. Phys.: Condens. Matter 16, L271 (2004); Phys. Rev. B 69, 241304(R) (2004).

${ }^{18}$ P. A. Maksym and T. Chakraborty, Phys. Rev. B 45, 1947 (1992).

${ }^{19}$ S. Tarucha, D. G. Austing, T. Honda, R. J. van der Hage, and L. P. Kouwenhoven, Phys. Rev. Lett. 77, 3613 (1996).

${ }^{20}$ C. Ellenberger, T. Ihn, C. Yannouleas, U. Landman, K. Ensslin, D. Driscoll, and A. C. Gossard, Phys. Rev. Lett. 96, 126806 (2006). 